\title{
LETTERS
}

Concise Research Reports

\section{A Randomized Trial to Improve Communication between Patients and Providers in a Primary Care Walk-In Clinic}

\author{
Jeffrey L. Jackson, MD, MPH \\ c, GIM Section, Zablocki VAMC, Medicine, Medical College of Wisconsin, Milwaukee, WI, USA.
}

$\mathrm{J}$ Gen Intern Med 33(4):404-5

DOI: $10.1007 / \mathrm{s} 11606-017-4300-y$

(C) Society of General Internal Medicine (outside the USA) 2018

\section{INTRODUCTION}

Effective communication is essential to high-quality care. ${ }^{1}$ In a previous pre-post trial among 500 adults presenting to a primary care walk-in clinic with a physical symptom, we provided pre-visit feedback to providers on patients' serious illness worry and visit expectations and the presence of any comorbid mental illness. ${ }^{2}$ Intervention patients had fewer unmet expectations and were less likely to be perceived as difficult by their physician. We found that unmet expectations were strong predictors of satisfaction, ${ }^{3,4}$ and worry correlated with psychiatric illness. ${ }^{5}$ The purpose of this trial was to replicate these findings using a more rigorous study design.

\section{METHODS}

We randomized adults presenting to a primary care walk-in clinic with a physical symptom (excluding upper respiratory illness, $n=187$ ) to two groups: usual care versus providers who received pre-visit information on patient serious illness worry, patient expectations for the visit (testing, counseling, diagnosis, treatment), and whether the patient had depression, anxiety or somatization. We used the same instruments and clinics as in our previous trial. ${ }^{2}$ Pre-visit, written patient surveys assessed symptom characteristics, illness worry, stress, functional status (Medical Outcomes Study [MOS] SF-6), depression (Patient Health Questionnaire [PHQ]-9), anxiety (7-item Generalized Anxiety Disorder scale [GAD-7]) and somatization (PHQ-15). Immediately post-visit patient surveys assessed satisfaction (VSQ-9) and trust (Trust in Physician-7), residual illness worry and unmet expectations. Surveys at 2 weeks assessed persistent worry, unmet expectations, satisfaction, functional status and symptom outcome; these surveys were mailed, with phone follow-up of non-responders. Immediate post-visit physician surveys assessed patient difficulty (Difficult Doctor-Patient Relationship Questionnaire [DDPRQ]). In addition, all providers attended a 2-h workshop on the importance of using this information to address worry, expectations and managing patients with psychiatric disorders. Providers were capped at 10 patients per

Published online February 5, 2018 arm. Our study was approved by the Walter Reed institutional review board (IRB). Sample size calculations were based on achieving the same reduction in patient difficulty as in our previous trial. $^{1}$

\section{RESULTS}

We enrolled 250 adults. Patients were managed by 24 primary care providers. There were no significant differences between arms (Table 1). Patients had experienced their symptoms for 14 days, and most (62\%) were worried that their symptoms could be serious. Illness worry declined immediately post-visit (25\%), though it rebounded to $41 \%$ by 2 weeks. Most patients had previsit encounter expectations. Our intervention had no impact on any outcome, including illness worry, unmet expectations, patient satisfaction either immediately post-

Table 1 Participant Characteristics

\begin{tabular}{|c|c|c|c|}
\hline & $\begin{array}{l}\text { Intervention } \\
(n=128)\end{array}$ & $\begin{array}{l}\text { Control } \\
(n=120)\end{array}$ & $P$ \\
\hline Age & 51.6 & 52.3 & 0.29 \\
\hline Female, $\%$ & $55 \%$ & $44 \%$ & 0.69 \\
\hline \multicolumn{4}{|l|}{ Ethnicity } \\
\hline White & $55(43 \%)$ & $54(45 \%)$ & \multirow[t]{4}{*}{0.70} \\
\hline Black & $54(42 \%)$ & $55(46 \%)$ & \\
\hline Asian & $9(7 \%)$ & $5(4 \%)$ & \\
\hline Hispanic & $6(5 \%)$ & $4(3 \%)$ & \\
\hline $\begin{array}{l}\text { Serious illness worry, } \\
\text { no. }(\%)\end{array}$ & $86(67 \%)$ & $70(58 \%)$ & 0.15 \\
\hline "Are you worried that your & & & \\
\hline $\begin{array}{l}\text { symptom could be due to a } \\
\text { serious illness?" }\end{array}$ & & & \\
\hline Stress, no. $(\%)$ & $59(46 \%)$ & $62(52 \%)$ & 0.38 \\
\hline $\begin{array}{l}\text { "In the past } 2 \text { weeks, have } \\
\text { you been experiencing } \\
\text { stress?" }\end{array}$ & & & \\
\hline Patient visit expectations & & & 0.99 \\
\hline Diagnosis & $101(79 \%)$ & $96(80 \%)$ & \\
\hline $\begin{array}{l}\text { Estimated duration of } \\
\text { symptom }\end{array}$ & $88(69 \%)$ & $79(66 \%)$ & \\
\hline Physical examination & $56(44 \%)$ & $56(47 \%)$ & \\
\hline Tests & $96(75 \%)$ & $82(68 \%)$ & \\
\hline Treatment & $65(51 \%)$ & $58(48 \%)$ & \\
\hline Referral & $51(40 \%)$ & $48(40 \%)$ & \\
\hline $\begin{array}{l}\text { Functional status } \\
\text { (MOS SF-6) }\end{array}$ & 22.2 & 22.5 & 0.60 \\
\hline Pain severity $(0-10)$, mean & 3.6 & 3.8 & 0.34 \\
\hline Symptom duration (median) & 15.5 & 14 & 0.83 \\
\hline $\begin{array}{l}\text { Somatization (PHQ-15, } \\
0-15)\end{array}$ & 3.6 & 3.9 & 0.92 \\
\hline $\begin{array}{l}\text { Mental illness (depression } \\
\text { or anxiety) }\end{array}$ & $44(28 \%)$ & $32(27 \%)$ & 0.83 \\
\hline
\end{tabular}


Table 2 Outcomes

\begin{tabular}{|c|c|c|c|}
\hline & $\begin{array}{l}\text { Intervention } \\
(n=128)\end{array}$ & $\begin{array}{l}\text { Control } \\
(n=120)\end{array}$ & $P$ \\
\hline \multicolumn{4}{|l|}{ Immediately post-visit } \\
\hline $\begin{array}{l}\text { Residual serious illness } \\
\text { worry, no. }(\%)\end{array}$ & $28(22 \%)$ & $35(29 \%)$ & 0.19 \\
\hline \multicolumn{4}{|c|}{ Unmet patient visit expectations } \\
\hline Diagnosis & $37(29 \%)$ & $40(33 \%)$ & 0.45 \\
\hline Estimated duration of & $59(46 \%)$ & $65(54 \%)$ & \\
\hline \multicolumn{4}{|l|}{ symptom } \\
\hline Physical examination & $11(9 \%)$ & $2(2 \%)$ & \\
\hline Tests & $9(7 \%)$ & $4(3 \%)$ & \\
\hline Treatment & $3(2 \%)$ & $0(0 \%)$ & \\
\hline Referral & $5(4 \%)$ & $6(5 \%)$ & \\
\hline Fully satisfied & $87(69 \%)$ & $70(70 \%)$ & 0.12 \\
\hline Patient considered & $19(15 \%)$ & $22(18 \%)$ & 0.46 \\
\hline \multicolumn{4}{|l|}{ "difficult" by provider } \\
\hline \multicolumn{4}{|l|}{ 2-week follow-up } \\
\hline \multicolumn{4}{|l|}{ Symptom outcome } \\
\hline Gone & $27(21 \%)$ & $26(22 \%)$ & 0.68 \\
\hline Better & $65(51 \%)$ & $54(45 \%)$ & \\
\hline Same & $29(23 \%)$ & $35(29 \%)$ & \\
\hline Worse & $5(4 \%)$ & $4(3 \%)$ & \\
\hline Residual serious illness & $49(38 \%)$ & $56(47 \%)$ & 0.18 \\
\hline worry & & & \\
\hline $\begin{array}{l}\text { Pain severity }(0-10) \text {, } \\
\text { mean }\end{array}$ & 3.4 & 3.5 & 0.84 \\
\hline Fully satisfied, no. (\%) & $76(63 \%)$ & $74(62 \%)$ & 0.71 \\
\hline $\begin{array}{l}\text { Functional status (MOS } \\
\text { SF-6) }\end{array}$ & 23.6 & 23.6 & 0.99 \\
\hline
\end{tabular}

visit or at 2 weeks, and 2-week symptom outcome (Table 2). There was also no difference between the groups in the likelihood of patients being labeled as difficult by their provider.

\section{DISCUSSION}

Our intervention had no impact on worry, satisfaction, functional status or symptom outcome over 2 weeks of follow-up. It also had no effect on the percentage of patients perceived as "difficult" by providers. While disappointing, our results are not surprising. One review of 35 randomized trials of interventions to alter patient-provider interactions found that provider behaviors were difficult to change, and most trials had little impact on patient outcomes. ${ }^{6}$
Moreover, our educational intervention was brief and our intervention was weak. It is possible that other factors played a larger role in outcomes or that there was a spillover effect, since both groups received the workshop. The previously observed improved patient outcomes are harder to explain, since patients did not know which arm they were in. However, it is common for randomized trials to see less impressive results than those in trials using quasi-experimental methods. Our previous trial results could have been due to chance, particularly given the large number of outcomes assessed. Improving patient-provider communication has proven to be an elusive goal.

Corresponding Author: Jeffrey L. Jackson, MD, MPH; C, GIM Section, Zablocki VAMC, Medicine Medical College of Wisconsin, Milwaukee, WI, USA (e-mail: jjackson@mcw.edu).

Funding No funding was received for this project.

\section{Compliance with Ethical Standards:}

Conflict of Interest: The author declares that he does not have a conflict of interest.

\section{REFERENCES}

1. Stewart MA, McWhinney JR, Buck CW. The doctor/patient relationship and its effect upon outcomes. J R Coll Gen Pract. 1979;29:77-81.

2. Jackson, JL, Kroenke, K, Chamberlin J. Effects of physician awareness of symptom-related expectations and mental disorders. a controlled trial. Arch Fam Med. 1999;8(2): 135-42.

3. Jackson JL, Chamberlin J, Kroenke K. Predictors of satisfaction. Soc Sci Med. 2001;52(4):609-20.

4. Jackson, JL, O'Malley PG, Kroenke K. Clinical predictors of mental disorders among medical outpatients, validation of the S4 model. Psychosomatics. 1998;39:431-6.

5. Jackson JL, Kroenke $\mathbf{K}$. The effect of unmet expectations among adults presenting with physical symptoms. Ann Intern Med. 2001;134:889-97

6. Griffin SJ, Kinmonth AL, Veltman MWM, Gillard S, Grant J, Stewart M. Effect on health-related outcomes of interventions to alter the interaction between patients and practitioners: a systematic review of trials. Ann Fam Med. 2004;2(6):595-608. 